\title{
REMOVAL OF THE THIRD THORACIC VERTEBRA AND PARTIAL LUNG RESECTION FOR A RADIORESISTANT GIANT-CELL TUMOUR OF THE SPINE
}

\author{
SVEN-ERIK LARSSON \\ From the Department of Orthopaedic Surgery, University of Umed, Sweden
}

\begin{abstract}
Total removal of the third thoracic vertebra and partial removal of the second and fourth vertebrae together with partial lung resection were successfully performed in a twenty-two-year-old woman with a large, radioresistant, giant-cell tumour which completely surrounded the spinal cord and extended over the left lung. On admission, the patient was in her third episode of paraplegia, the two previous episodes having been temporarily relieved after decompression of the spinal cord by laminectomy and partial removal of the tumour.

Three and a half months after operation she was discharged walking without support and with normal sphincter control. Two years later she is free of symptoms and the neurological status is practically normal. Clinical and radiological examinations show no signs of recurrence of the tumour.
\end{abstract}

Total removal of a vertebra, spondylectomy, was described in 1968 by Lièvre and his collaborators who, in a two-stage operation, removed the fourth lumbar vertebra which was the site of a giant-cell tumour: the posterior parts were removed first and then after two weeks the body of the vertebra (Lièvre et al. 1968). Removal of the second lumbar vertebra because of a malignant tumour was reported by Hamdi in 1969. Complete removal of three vertebrae (the eleventh and twelth thoracic and first lumbar) was described by Stener and Johnsen (1971), who after a laminectomy removed a large giant-cell tumour together with all remaining parts of the three adjacent vertebrae. In another case a chondrosarcoma arising from the seventh thoracic vertebra was excised with total removal of the seventh and partial removal of the sixth and eighth thoracic vertebrae (Stener 1971).

This report describes total removal of the third and partial removal of the second and fourth thoracic vertebrae together with partial lung resection in a twenty-two-year-old woman with a radioresistant giant-cell tumour arising from the third thoracic vertebra. The reason that total spondylectomy at this level has not been described earlier could be the high risk of producing a paraplegia by damaging the nutrition of the spinal cord at operation. The nutrition is dependent upon "radicular" arteries accompanying the nerves to the cord; the critical level shows individual variation but is usually in the region of the third thoracic vertebra, according to Lazorthes et al. (1958). Further, the width of the thorax is so limited at this level that great difficulties are experienced in the surgical exploration of the spine.

\section{CASE REPORT}

The patient, aged twenty-two years, was admitted to the department of neurosurgery in Uppsala on February 20, 1975. For three months she had suffered gradually increasing pain in the interscapular region radiating somewhat to the left. On February 2 she experienced inconspicuous fasciculations in the right leg and a few days later in the left leg. The week before admission she had had difficulties in walking and had fallen several times because of stiffness, weakness and unsteadiness of both legs. On admission she could only walk a few steps with support and also had difficulty with micturition. Physical examination showed a spastic paralysis of the lower limbs with no active movements at either ankle. Ankle clonus and Babinski's sign were present bilaterally. Sensory loss was noted on both sides up to the level of T6. Radiographs and tomograms showed a destruction of the left part of the body of the third thoracic vertebra and the left pedicles of T3 and T4. She was fifteen weeks pregnant.

Decompressive laminectomy of $\mathrm{T} 3$ and $\mathrm{T} 4$ was immediately performed with a biopsy of the tumour which was causing compression of the spinal cord at the level of the third thoracic vertebra. Histopathological examination showed a giant-cell tumour of bone with atypical stromal cells corresponding to Grade III in the classification of Jaffe, Lichtenstein and Portis (1940). After operation she was free of pain and the paraparesis gradually regressed. Two weeks later her pregnancy was terminated. During the next three months high-voltage radiotherapy was given to the third and fourth thoracic vertebrae.

In December 1975 she was readmitted for acute, complete paraplegia below the level of T3. At operation the spinal cord was found to be riding over the upper part of the body of the fourth thoracic vertebra because of collapse of T3. Tumour tissue was present on both sides of the spinal cord. Decompression of the spinal cord was carried out from T2 to T5. Histopathological examination of a biopsy specimen showed the same picture as that of the first examination. After operation there was again good regression of the paraplegia. She was able to walk without support and could move her legs normally.

In January 1977 she was admitted to the department of orthopaedic surgery in Umeå. During the previous four weeks she had experienced recurrent symptoms of progressively increasing paraplegia. Neurological examination showed a spastic paraplegia with 
bilateral ankle and patcllar clonus and positive Babinski's sign. She was again unable to walk and complained of frequent micturition. The grouing tumour was now visible to the left of the thoracic spine (Fig. 1). Radiographs showed almost complete destruction of the body of T3 with a pronounced kyphosis (Figs. 2 and 3). There were no pulmonary metastases but the tumour had spread from T3 and extended from the skin of the back over to the mediastinum and the apical part of the left lung, where it measured about $4 \times 5$ centimetres. and completely surrounded the spinal cord (Figs. 4 and 5). In view of the compression of the spinal cord by the tumour and the progressing kyphosis, aortography was not performed because of the risk of causing paraplegia.

The patient was now suffering from progressive symptoms of spastic paraplegia, recurring for the third time. She had received modern. high-voltage radiotherapy at an accumulated dose just below the limit for spinal cord damage. Despite the optimal radiotherapy, there wa's evident progress of the tumour. Paraplegia and death could be expected. It was therefore decided to attempt extirpation of the tumour.

Because of the site and spread of the tumour it had to be removed in one operation involving three stages. First, all the posterior part of the tumour had to be removed together with the parts that had destroyed the pedicles and surrounded the spinal cord on both sides. Then partial lung resection and removal of all the anterior part of the tumour had to be performed via a transthoracic approach, opening the mediastinum from the left side. Finally, the spine had to be reconstructed, first by inlaying a large bone block from the iliac crest between the remaining parts of the bodies of $\mathrm{T} 2$ and $\mathrm{T} 4$ and then by stabilisation of the spine with two A.O. plates of stainless steel fixed with wires to the transverse processes of the vertebrae situated above and below the space left by the removal of T3. A plaster bed was prepared to be used for a period of three months after operation. The thoracotımy was performed with the participation of Dr Adar Hallén, M.D., from the Department of Thoracic Surgery, University Hospital, Uppsala. Sweden.

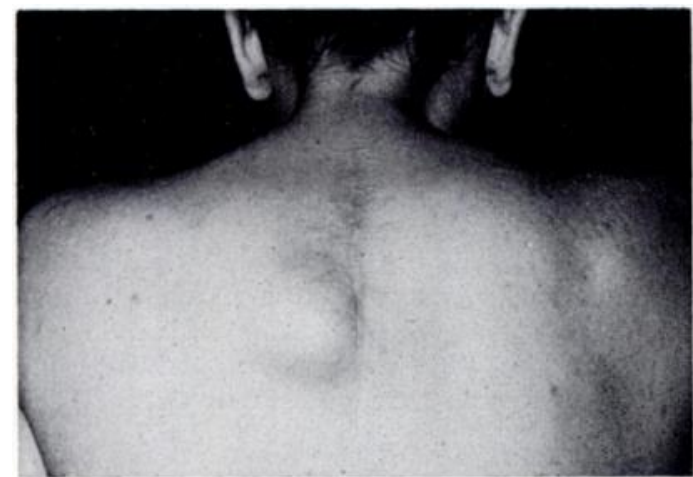

Fig. 1

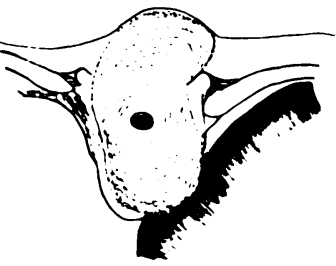

Fig. 4

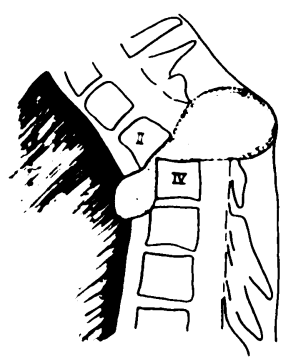

Fig. 5

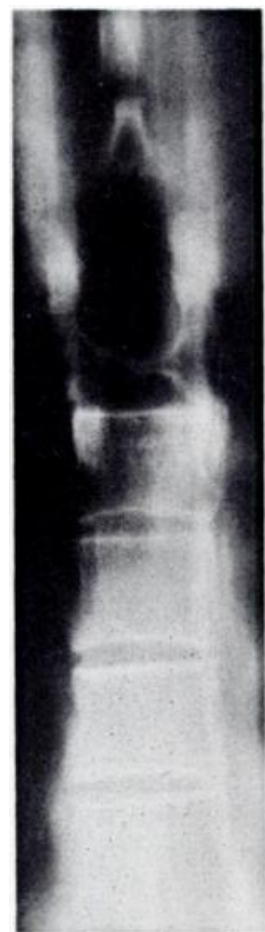

Fig. 2

\section{OPERATION}

The patient was placed prone on a Wilson frame in order to aveid compression of the vena cava. A midline incision over the thoracic spine as far as T8 was extended to the left along the eighth rib. The well-delimited. posterior part of the tumour measuring about $8 \times 10$ centimetres was removed. The spinal cord was then exposed and all tumour tissue carefully removed together with the scar tissue from T2 to T5 which had formed after the two previous operations (Fig. 6). Due to a kyphosis of about 70 degrecs the spinal cord was stretched over the upper posterior part of the body of T4. The spinal cord and the surrounding thecal sac were not infiltrated by the tumour. The part of the tumour surrounding the spinal cord on the right side was removed after resection of the posterior 5 centimetres of the third rib together with the transverse process of $\mathrm{T} 3$, the right pedicle and adjacent parts of the vertebral body.

The operation was then continued within the thorax. The patient was placed on her right side and a left thoracotomy incision made between the fifth and sixth ribs. The tumour had infiltrated into the left thoracic wall and the apical part of the upper lobe of the left lung (Fig 7), where it was well delimited and could be removed by a radical excision leaving a defect in the thoracic wall (Fig. 8). The remaining part of the tumour within the body of the third vertebra could then be removed by resecting parts of the vertebral bodies of $\mathrm{T} 2$ and $\mathrm{T} 4$. All the tumour had now been removed. This left a gap in the thoracic spine which was bridged only by the spinal cord enclosed in the thecal sac. The head and shoulders of the patient were held by an assistant so that the spinal cord was not stretched or twisted.

For the reconstruction of the spine, a tensor instrument was placed between the remaining parts of $\mathrm{T} 2$ and $\mathrm{T} 4$. Then two blocks of bone, each about 3 centimetres long. were taken from the iliac crest. placed within the gap between $\mathrm{T} 2$ and $\mathrm{T} 4$ and the tensor removed. It was not possible to achieve full correction of the kyphosis because of the risk of stretching the spinal cord. The tension of the large muscles tended to maintain the kyphosis. For internal fixation of the spine, two A.O. plates with ten holes were used, each measuring $18 \times 1.6 \times 0.6$

Figure 1-The giant-cell tumour arising from the body of the third thoracic vertebra is seen protruding to the left of the scar from two previous decompression operations. Figures 2 and 3-Anteroposterior and lateral tomograms showing the collapsed body of the third thoracic vertebra and the defect after previous laminectomies. Figures 4 and 5 -Diagrams of horizontal and lateral sections to show the extent of the tumour. 


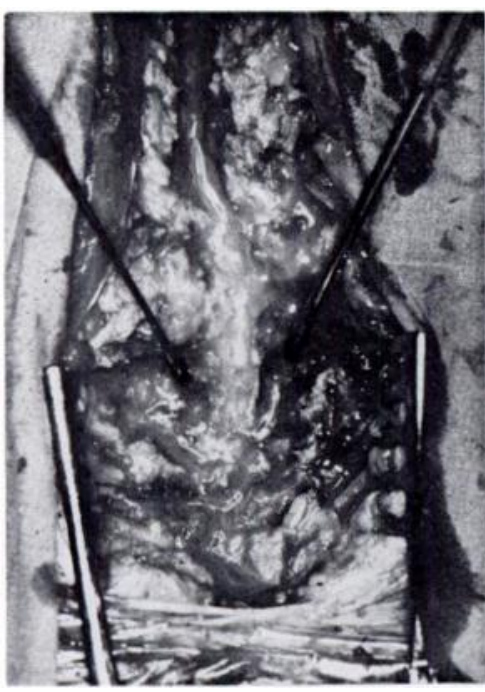

Fig. 6

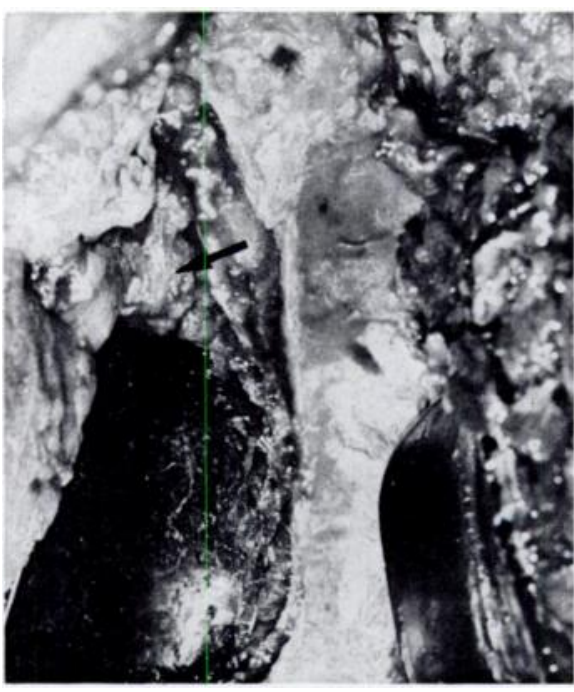

Fig. 7

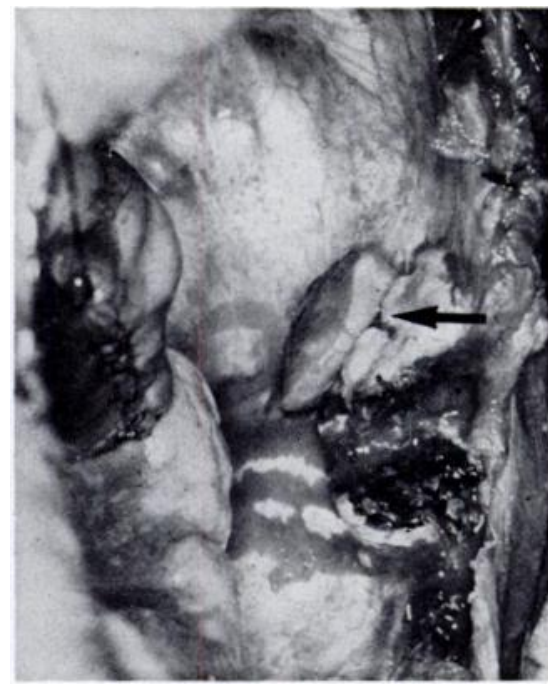

Fig. 8

Figure 6-The spinal cord has been exposed after removal of the part of the tumour which surrounded the posterior part of the cord. The dissectors show the tumour on both sides of the spinal cord. Figure 7-After thoracotomy, the tumour (arrow) can be seen infiltrating through the thoracic wall to the left lung. Figure 8-The part of the tumour involving the left lung has been removed and the resected lung sutured (left). The remaining part of the tumour is seen to the right (arrow).

centimetres (Figs. 9 and 10). First. one plate was wired to the left transverse processes of vertebrae T1, T2, T5, T6 and T7. Thereafter the defect of the posterior thoracic wall was closed with a thoracoplasty. One suction tube was inserted into the left pleural cavity posteriorly, the thoracotomy wounds were closed and the muscles which had been detached were reattached to the spine. Then the patient was again placed prone and the other plate was wired to the right transverse processes of the same vertebrae as the first plate. There were difficulties in achieving a firm fastening of the plates to the proximal part of the divided spine because transverse processess were

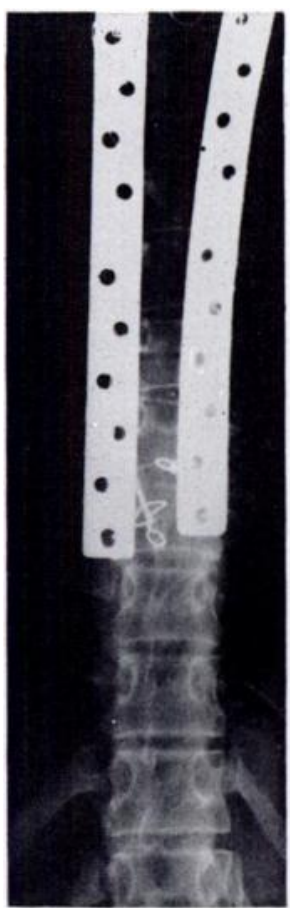

Fig. 9

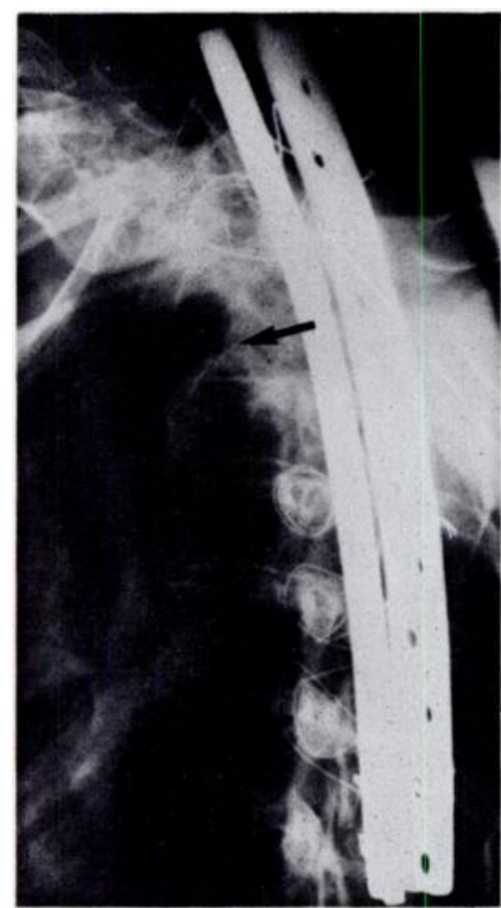

Fig. 10
After complete removal of the tumour, including the whole third thoracic vertebra and parts of the second and fourth, the gap has been bridged by two bone blocks (arrow) taken from the iliac crest. Stabilisation of the spine was accomplished with two steel plates fixed with wires to the transverse processes. only available at two levels. The spinal cord was intact and without any persisting signs of distraction or compression. Finally the muscles on the right side were reattached to the spine and the skin was closed with steel sutures. Operating time was thirteen and a half hours and the total blood loss was 13500 millilitres. The patient was in good condition throughout the operation, and was taken out of the respirator twelve hours later.

Operative specimen. The large reddish-brown tumour was examined by $\operatorname{Dr}$ L. Boquist who reported histopathological appearances characteristic of Grade III giant-cell tumour. There was a good

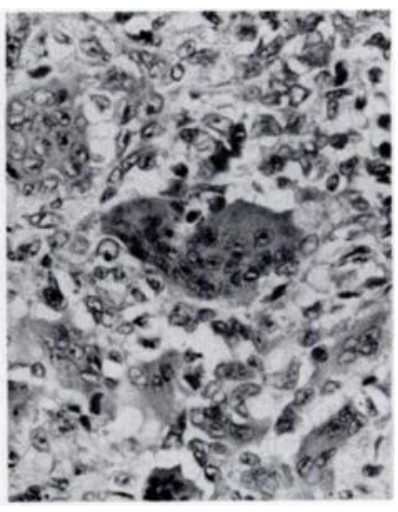

Fig. 11

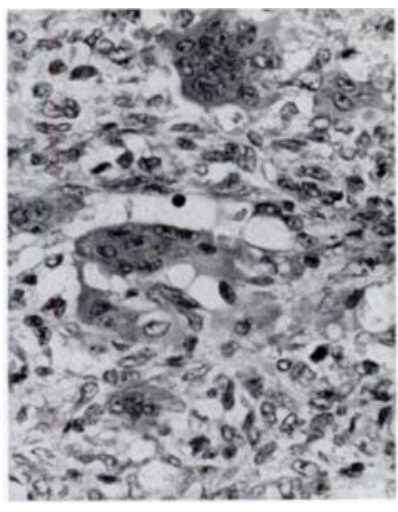

Fig. 12
Photomicrographs of the giant-cell tumour showing oval or spindleshaped stromal cells with atypical, irregular hyperchromatic nuclei and mitotic figures. Giant cells of varying size can be seen with numerous nuclei and distinct nucleoli.

correspondence between the initial and final histopathological appraisals of the lesion with regard to the degrec of malignancy. The tumour was composed of stromal cells, giant cells, and occasional collagenous fibrils. Some tumour cells were atypical and had irregular hyperchromatic nuclei (Figs. 11 and 12) and mitotic figures. There were areas of bone destruction that also contained regions of fibrosis. haemorrhage, and cyst formation.

\section{PROGRESS}

After operation the patient was nursed in a plaster bed which was tilted 20 degrees to the left and right at regular intervals. Oral feeding was started after two days, the pleural tube was removed after three days and the urethral catheter after six days. She was given anticoagulants 


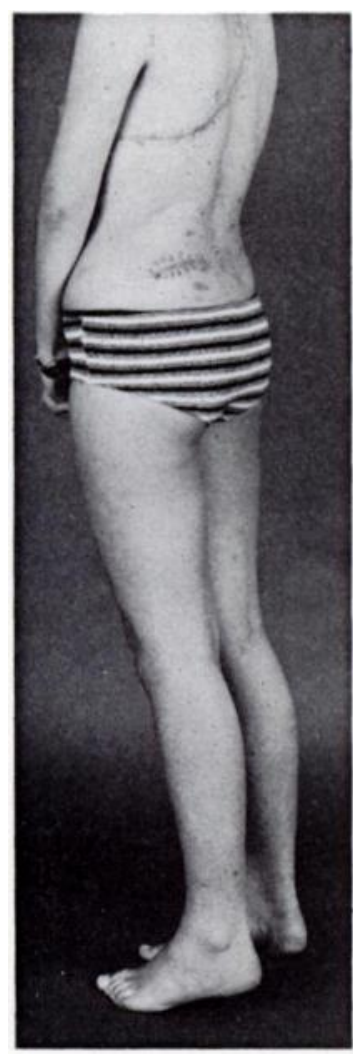

Fig. 13

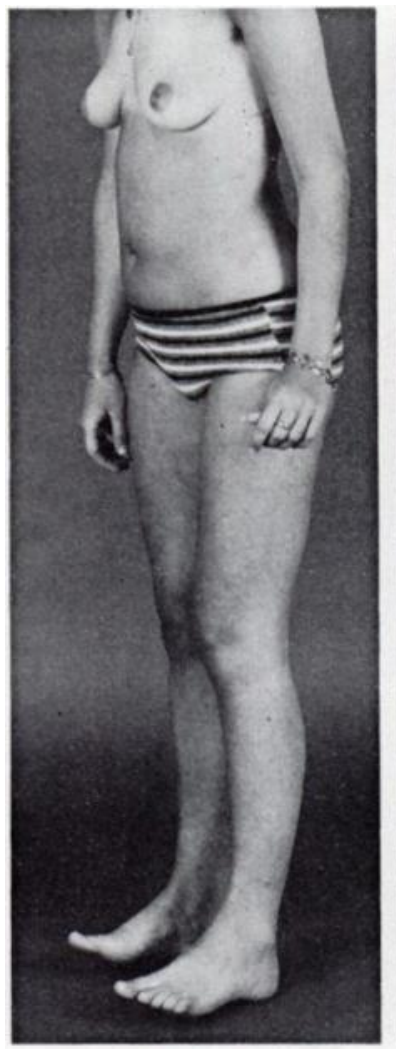

Fig. 14

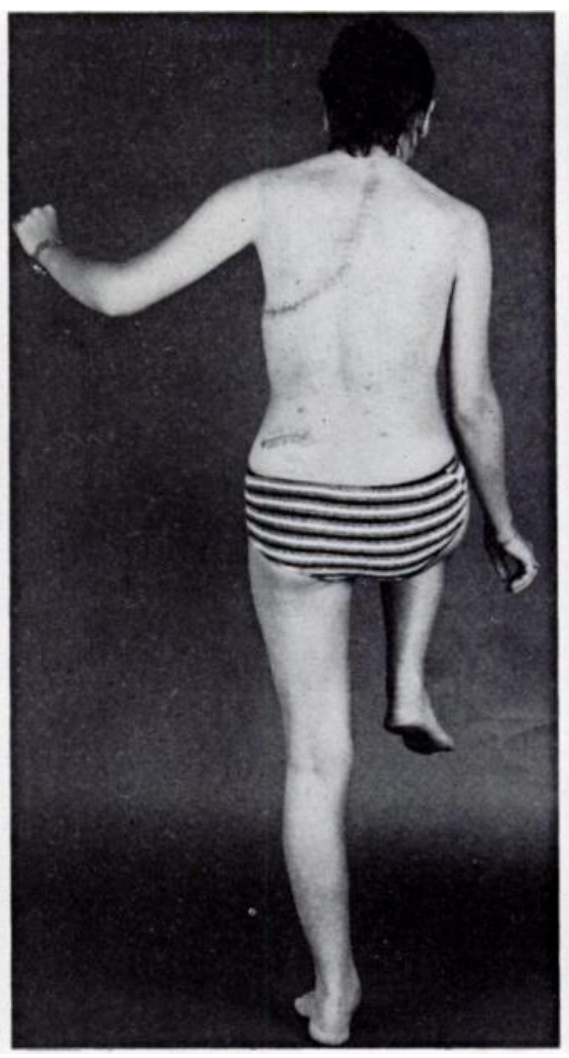

Fig. 15

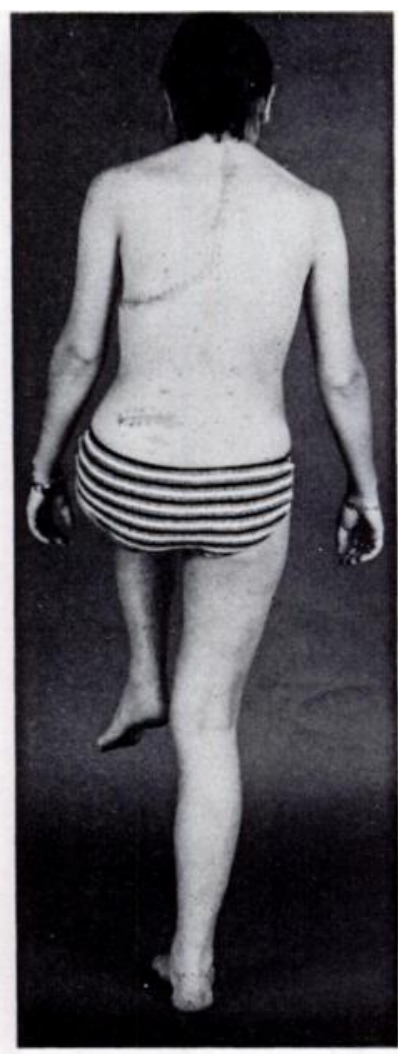

Fig. 16

Physical ability eight months after operation showing standing on toes and heels, negative Trendelenburg's sign and good active movement of the spine.

and prophylactic antibiotics. All wounds healed by first intention and there were no pressure sores. The paraplegia and spasticity gradually regressed.

Three months after operation gradual training to an upright position was started with the aid of a tilting table. About two weeks later she was allowed to start walking without external support. On April 28, 1977, after three and a half months in hospital she was discharged home.
Eight months after operation she was readmitted for removal of the plates. She had no neurological symptoms except for a slight weakness of the left leg apparent after 2000 metres of rapid walking. She walked without limping, could stand normally on her toes or heels or on either leg alone, and her spine showed good active movement except for a persisting kyphosis at T3 (Figs. 13 to 16). Sphincter functions were normal.

Now, two years after the operation, her physical condition has

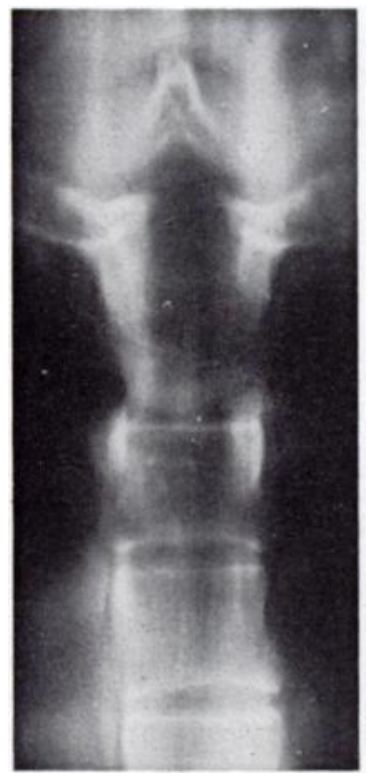

Fig. 17

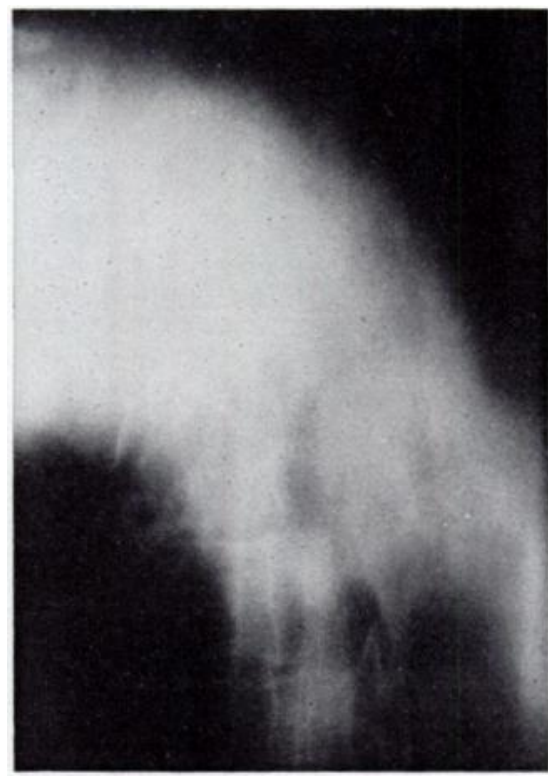

Fig. 18

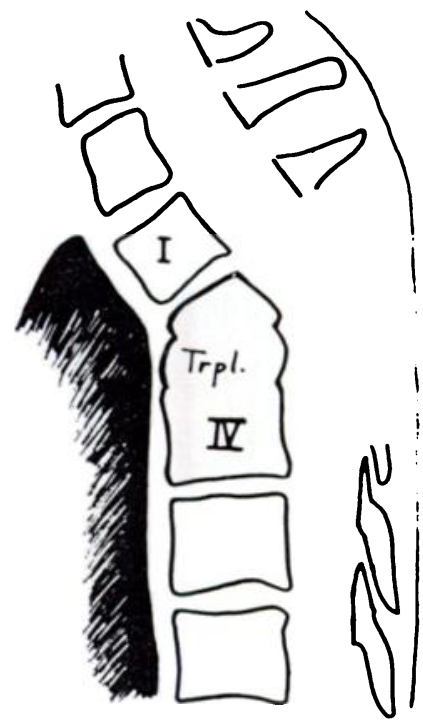

Fig. 19

Figures 17 and 18-Tomograms of the spine after removal of the plates eight months after operation showing fusion between the iliac grafts and the bodies of the second and fourth thoracic vertebra, as illustrated in Figure 19. 
improved further. She has no spinal complaints, is walking normally and has started to play tennis. Neurological status is normal except for slightly exaggerated reflexes of the lower limbs. Clinical and radiological examinations show no recurrence of the the tumour. Radiographic progress. On removal of the plates eight months after operation tomography showed that fusion had taken place between the iliac grafts and the bodies of the second and fourth thoracic vertebra, to form a bony block (Figs. 17 and 18). The grafts have healed with reduced height anteriorly causing a kyphosis of 55 degrees (Fig. 19). The present radiographs have a similar appearance with no signs of recurrence of the tumour.

\section{DISCUSSION}

Total removal of the third thoracic vertebra has not been described before, presumably because of the high risk of failure. It had to be undertaken in this young patient with a spinal tumour because of recurrent paraplegia and risk to life. A giant-cell tumour of the spine is rare and there are only a few cases reported in the literature. In a previous clinical and histopathological study, four patients with a tumour of the spine and one with a sacral tumour were reported (Larsson, Lorentzon and Boquist 1975a). These were all Grade I tumours histopathologically, and showed good prognosis after high-voltage radiotherapy together with partial removal of the tumour. The total spondylectomy by Stener and Johnsen (1971), performed on a twenty-six-year-old woman with a giant-cell tumour arising from the twelfth thoracic vertebra, was not appraised histopathologically for the degree of malignancy. Radiotherapy was not given as the tumour was considered to be operable. The present case was a Grade III tumour which had proved resistant to modern high-voltage radiotherapy. The alternative to operation would have been cytostatic treatment from which only a palliative effect, if any, could have been anticipated.

The histopathological grading of giant-cell tumours is generally of little prognostic value (Larsson et al. $1975 \mathrm{~b})$. Eleven per cent of all giant-cell tumours of bone are clinically malignant. A mortality of 36 per cent was found for the tumours located within the distal femur (Larsson et al. 1975a). In view of the atypical cellular structure, the mitotic activity, the infiltrative growth pattern and the radioresistance of the present spinal tumour, this neoplasm was obviously malignant.

At present, more than two years after the operation, the patient is free of any symptoms and shows no radiographical signs of recurrence of the tumour. Its radical removal and the fact that 97 per cent of the recurrencies occur within two years (Goldenberg, Campbell and Bonfiglio 1970) should mean that she has a good chance of long survival.

The method used for reconstruction of the spine after extirpation of the tumour was essentially that described by Stener (1971). Proximally, only the transverse processes of the first and second thoracic vertebrae could be used for fastening the plates. A somewhat limited internal fixation of the proximal part of the spine was therefore obtained. The muscle contracture which was secondary to the long-standing kyphosis contributed to the difficulties of achieving a full correction of the deformity. The persisting kyphosis of 55 degrees does not seem to be sufficient to create a risk of myelopathy.

This work was supported by grants from the Swedish Cancer Society No. 711-B78-06X.

\section{REFERENCES}

Goldenberg, R. R., Campbell, C. J., and Bonfiglio, M. (1970) Giant-cell tumor of bone: an analysis of two hundred and eighteen cases. Journal of Bone and Joint Surgery, 52-A, 619-664.

Hamdi, F. A. (1969) Prosthesis for an excised lumbar vertebra: a preliminary report. Canadian Medical Association Journal, 100, 576-580.

Jaffe, H. L., Lichtenstein, L., and Portis, R. B. (1940) Giant cell tumor of bone. Its pathologic appearance, grading, supposed variants and treatment. Archives of Pathology, 30, 993-1031.

Larsson, S.-E., Lorentzon, R., and Boquist, L. (1975a) Giant-cell tumor of bone. A demographic, clinical and histopathological study of all cases recorded in the Swedish Cancer Registry for the years 1958 through 1968. Journal of Bone and Joint Surgery, 57-A, 167-173.

Larsson, S.-E., Lorentzon, R., and Boquist, L. (1975b) Giant-cell tumors of the spine and sacrum causing neurological symptoms. Clinical Orthopaedics and Related Research, 111, 201-211.

Lazorthes, G., Poulhes, J., Bastide, G., Roulleau, J., and Chancholle, A.-R. (1958) La vascularisation artérielle de la moelle. Neuro-Chirurgie, 4, 3-19.

Lièvre, J.-A., Darcy, M., Pradat, P., Camus, J.-P., Bénichou, C., Attali, P., and Joublin, M. (1968) Tumeur a cellules géantes du rachis lombaire, spondylectomie totale en deus temps. Revue du rhumatisme et des maladies ostéo-articulaires, 35, 125-130.

Stener, B. (1971) Total spondylectomy in chondrosarcoma arising from the seventh thoracic vertebra. Journal of Bone and Joint Surgery, 53-B, 288-295.

Stener, B., and Johnsen, O. E. (1971) Complete removal of three vertebrae for giant-cell tumour. Journal of Bone and Joint Surgery, 53-B, 278-287. 